\title{
LOCALLY MAXIMAL BEZOUT DOMAINS
}

\section{SYLVIA WIEGAND}

ABSTRACT. Let $X$ be a finite tree. It is shown that $X$ is orderisomorphic to the prime spectrum of a Bezout domain $R$ such that every localization of $R$ is a maximal valuation ring.

Let $R$ be a Bezout domain and let spec $R$ denote the set of prime ideals of $R$ considered as a partially ordered set under inclusion. It is well known that the localizations of $R$ at prime ideals are valuation rings and that $\operatorname{spec} R$ is a tree: for each $s \in \operatorname{spec} R,\{x \in \operatorname{spec} R: x \leq s\}$ is totally ordered. The trees of the form spec $R, R$ a Bezout domain, have been completely characterized by Lewis [L], although the localizations $R_{M}$ in this construction are in general badly behaved. In this paper we show that every finite tree with a unique minimal element is order-isomorphic to spec $R$, for some Bezout domain $R$ which is locally a maximal valuation ring.

The construction in this paper is a generalization of an example of Barbara Osofsky which appears in [M2]. I would like to thank her for sending me the details of her construction with some suggestions for generalization. Also I am grateful to Tom Shores who carefully worked out some helpful facts about maximally complete fields.

Main theorem. Let $X$ be a finite tree. Then there exists a Bezout domain $R$ such that (1) spec $R$ is order-isomorphic to $X$, and (2) the localizations of $R$ at prime ideals are maximal valuation rings.

Proof. The procedure will be to exhibit $R$ as the intersection of a finite number of maximal valuation rings. Let $\mathcal{C}=\left\{C_{0}, C_{1}, \cdots, C_{n}\right\}$ denote the set of maximal chains indexed so that length $\left(C_{i}\right) \leq$ length $\left(C_{i-1}\right), 1 \leq i \leq$ $n$. (As usual, the length of a chain is one less than its cardinality.) For each maximal chain $C_{i}$, we find a maximal valuation ring $\Theta_{i}$, whose rank is equal to the length of the chain $C_{i}$ and whose quotient field is an alge-

Received by the editors November 6, 1973.

AMS (MOS) subject classifications (1970). Primary 13F05; Secondary 13A15, $13 \mathrm{C} 05$. 
braically closed field $K$ (fixed for all $i$ ). More precisely, we first construct $\Theta_{0}$, whose rank is equal to the length of $C_{0}$; then each $\mathcal{O}_{i}$ will be an appropriate localization of $\Theta_{0}$. In order to produce maximal valuation rings $V_{i}, 0 \leq i \leq n$, such that $R=V_{0} \cap V_{1} \cap \cdots \cap V_{n}$ has the correct prime spectrum, we will define automorphisms $\alpha_{i}$ of $K$ in such a way that $V_{i}=\alpha_{i}\left(\Theta_{i}\right)$ has the appropriate number of prime ideals in common with $\Theta_{0}$.

In what follows, we let $\mathbf{C}$ denote the field of complex numbers; $\mathbf{Q}$ is the additive ordered group of the rationals; and $\mathbf{Q}^{+}$is the set of positive rationals. Let $k$ be the length $\left(C_{0}\right)$, a longest chain in $X$.

We begin by constructing $\mathcal{O}=\mathcal{O}_{0}$, which is to be a valuation ring of rank $k$. Define a valuation $v$ on $\mathrm{C}\left(x_{1}, x_{2}, \cdots, x_{k}\right)$, the quotient field of the complex numbers, with $k$ indeterminates adjoined, to be the valuation induced by $v\left(x_{1} x_{2} x_{2} \ldots x_{k}^{a_{k}}\right)=\left(a_{1}, a_{2}, \cdots, a_{k}\right) \in G$, where $G$ is the direct sum of $k$ copies of $\mathbf{Q}$, ordered lexicographically. Let $K$ be the maximal completion of $\mathbf{C}\left(x_{1}, x_{2}, \cdots, x_{k}\right)$ with respect to $v$; we denote the extension of $v$ to $K$ by $v$ also. Now $\Theta$, the valuation ring of $K$, is a maximal valuation ring since $K$ is maximally complete [S, p. 45]. Also $K$ is algebraically closed. (The latter statement follows from [S, Theorem 12 , p. 57 and Theorem 11, p. 54].)

Since $v$ behaves properly on monomials with integer exponents, it must do so on monomials with rational exponents, that is $v\left(x_{1}{ }_{1} x_{2}{ }_{2} \ldots x_{k}{ }^{k}\right)=$ $\left(a_{1}, a_{2}, \cdots, a_{k}\right)$, for all $a_{i} \in \mathbf{Q}^{+}$. Now for each $j \leq k, P_{j}=\left\{r x_{j}^{a} \mid r \in \mathcal{O}\right.$, $\left.a \in \mathbf{Q}^{+}\right\}$is a prime ideal of $\Theta$. (Note that an element $r \in \mathcal{O}$ is in $P_{j}$ if and only if $v(r)$ has one of its first $r$ coordinates positive.) Also, because the rank of $G$ is $k$, these together with $P_{0}=(0)$ are all the prime ideals of 0 . (See [S, Corollary, p. 15] for more details here.) To see that $P_{j-1} \subseteq$ $P_{j}$, note that $v\left(x_{j-1} / x_{j}\right)=(0,0,0, \cdots, 0,1,-1,0,0, \cdots, 0)$, which is positive in the lexicographic ordering. Thus $x_{j-1} / x_{j} \in \mathcal{O}$, so $x_{j-1}^{a} \in P_{j}$ for all $1 \leq j \leq k$ and $a \in \mathbf{Q}^{+}$.

Thus we have found a valuation ring $O$ with quotient field $K$ and valuation $v: K \rightarrow G$ (the direct sum of $k$ copies of $\mathbf{Q}$ ), satisfying these properties:

(1) $K$ contains $\mathrm{C}\left(x_{1}, x_{2}, \cdots, x_{k}\right)$, where $x_{1}, x_{2}, \cdots x_{k}$ are indeterminates over $C$.

(2) The map $v: K \rightarrow G$ reads exponents of monomials, that is, for any set of $k$ rational numbers $\left\{a_{1}, a_{2}, \cdots, a_{k}\right\}$, we have $v\left(x_{1}{ }_{1} x_{2}{ }_{2} \cdots x_{k}{ }^{k}\right)=$ $\left(a_{1}, a_{2}, \cdots, a_{k}\right)$.

(3) The prime ideals of $\mathcal{O}$ are $\left\{P_{j} \mid 0 \leq j \leq k\right\}$ where $P_{0}=(0)$ and 
$P_{j}=\left\{r x_{j}^{a} \mid r \in \mathcal{O}, a \in \mathbf{Q}^{+}\right\}$; also $P_{i} \subseteq P_{j}$ if $i \leq j$.

We now pause to establish notation which will be fixed for the rest of the paper. As above, suppose (i) $X$ has maximal chains $C_{0}, C_{1}, \cdots, C_{n}$, (ii) for each $0 \leq i \leq n$, the length of $C_{i}$ is $k_{i}$ (a positive integer), and (iii) $k_{0} \geq k_{1} \geq \cdots \geq k_{n^{*}}$. Write (iv) $C_{i}=\left\{c_{i 0}, c_{i 1}, \cdots, c_{i k_{i}}\right\}$, where $C_{i 0}<c_{i 1}<$ $\cdots<c_{i k_{i}}$.

We use an induction procedure to define automorphisms $a_{i}$ of $K$. Let $\alpha_{0}$ be the identity automorphism. To define $\alpha_{i}$, choose some maximal chain $C_{m}$ with $0 \leq m \leq i-1$, such that among all the maximal chains $C_{0}, C_{1}, \cdots$, $C_{i-1}$, the chain $C_{m}$ has the largest number of elements in common with $C_{i}$. (Clearly the minimal element of $X$ is in every $C_{i}$.) Let $r$ be the (unique) integer such that $c_{i j}=c_{m j}$ for all $j<r$ but $c_{i r} \neq c_{m r}$. (Then, since $X$ is a tree, $c_{i j} \neq c_{m j}$ for all $r \leq j \leq k_{m}$.) Define $\alpha_{i}$ by $\alpha_{i}\left(x_{j}\right)=$ $a_{m}\left(x_{j}\right)$ if $0<j<r$ and $a_{i}\left(x_{j}\right)=x_{j}+i$ if $r \leq j \leq k$, and extend $a_{i}$ to an automorphism (still called $a_{i}$ ) of $K$ fixing $C$. (Here $i$ is regarded as a constant polynomial.) This is possible since the $x_{j}$ are part of a transcendence basis for $K$ over $\mathbf{C}$, and $K$ is algebraically closed. Notice that the $a_{i}$ have been defined so that

(4) For each $j$, if $m$ is the least integer for which $c_{m j}=c_{i j}$, then $a_{i}\left(x_{j}\right)=a_{m}\left(x_{j}\right)=x_{j}+m$. In particular $a_{i}\left(x_{k_{i}}\right)=x_{k_{i}}+i$.

(5) Claim: If $a_{i}\left(x_{l}\right) \in \alpha_{m}\left(P_{t}\right)$, for $0 \leq i, m \leq n$ and $1 \leq l, t \leq k$, then $a_{i}\left(x_{l}\right)=a_{m}\left(x_{l}\right)$ and $l \leq t$.

Proof. By (4) above, $a_{i}\left(x_{l}\right)=\alpha_{m}\left(x_{l}\right)+d$, for some integer $d$. Thus $a_{m}\left(x_{l}+d\right)=a_{m}\left(x_{l}\right)+d=a_{i}\left(x_{l}\right) \in a_{m}\left(P_{t}\right)$, and so $x_{l}+d \in P_{t}$. If $d \neq$

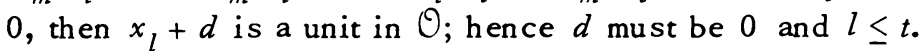

Let $\mathcal{O}_{i}$ be the localization $\mathcal{O}_{P}$ for $0 \leq i \leq n$, and let $V_{i}=\alpha_{i}\left(\Theta_{i}\right)$. Our next project is to investigate the prime ideals of $R=V_{0} \cap V_{1} \cap \cdots \cap V_{n}$.

Notice that the $V_{i}$ are irredundant in the intersection for $R$. For if not, suppose $V_{0} \cap V_{1} \cap \cdots \cap V_{m-1} \cap V_{m+1} \cap \cdots \cap V_{n} \subseteq V_{m}$. Let $t=k_{m}$ and set $N_{m}=$ $a_{m}\left(P_{t}\right)$, the maximal ideal of $V_{m}$. Now set $z=x_{t}+m=\alpha_{m}\left(x_{t}\right) ; z$ is an element of $N_{m}$, so $1 / z \notin V_{m}$. Thus $1 / z \notin V_{i}$ for some $i \neq m$, whence $z \epsilon$ $N_{i}=\alpha_{i}\left(P_{k_{i}}\right)$, the maximal ideal of $V_{i}$. By $(5), \alpha_{m}\left(x_{t}\right)=\alpha_{i}\left(x_{t}\right)$ and $t \leq$ $k_{i}$, and using (4) we deduce $c_{m t}=c_{i t}$. Since $X$ is a tree, $c_{m j}=c_{i j}$ for all $0 \leq j \leq t$. Now $C_{m}$ has only $t$ el ements; thus $C_{m} \subseteq C_{i}$, a contradiction.

By [K, Theorem 107, p. 78], $R$ is Bezout and the maximal ideals of $R$ are precisely $\left\{N_{j} \cap R, 0 \leq j \leq n\right\}$, where $N_{j}$ is the maximal ideal of $V_{j}$; also $V_{j}$ is the localization of $R$ at $N_{j} \cap R$. It easily follows that every prime ideal of $R$ is of the form $Q \cap R$, where $Q$ is a prime ideal of some $V_{j}$, that is: 
(6) The prime ideals of $R$ are of the form $\alpha_{i}\left(P_{j}\right) \cap R$ with $0 \leq i \leq n$, $0 \leq j \leq k_{i}$. (Note that these ideals need not be distinct for different $i$. )

Each localization of $R$ is a localization of some $V_{i}$ and the latter is isomorphic to the maximal valuation ring $\Theta_{i}$. Therefore the localizations of $R$ at prime ideals are maximal valuation rings.

To complete the proof, we define a function $\phi$ from $X$ to spec $R$ by $\phi\left(c_{i j}\right)=\alpha_{i}\left(P_{j}\right) \cap R$; we will show $\phi$ is an order isomorphism. First we check well-definedness of $\phi$. It suffices to show that if $c_{l j}=c_{s t}$ for some $s<l$, then $\phi\left(c_{l j}\right)=\phi\left(c_{s t}\right)$. Since $X$ is a tree, $j=t$ and $c_{l h}=c_{s h}$ for all $h \leq j$. As in (4), let $m$ be the smallest nonnegative integer so that $c_{m j}=c_{l j}$; then $\alpha_{m}\left(x_{j}\right)=\alpha_{l}\left(x_{j}\right)$ and so $\alpha_{m}\left(P_{j}\right)=\alpha_{l}\left(P_{j}\right)$. Also $m \leq s$ and $m$ is the smallest index with $c_{m j}=c_{s j}$, so $\alpha_{m}\left(P_{j}\right)=\alpha_{s}\left(P_{j}\right)$. Thus $\phi\left(c_{l j}\right)=\phi\left(c_{s t}\right)$.

The function $\phi$ is an isomorphism. For suppose $\phi\left(c_{i j}\right)=\phi\left(c_{l t}\right)$, that is $a_{i}\left(P_{j}\right) \cap R=a_{l}\left(P_{t}\right) \cap R$, then $\alpha_{l}\left(x_{t}\right) \in \alpha_{i}\left(P_{j}\right) \cap R$. By (5), this implies $\alpha_{i}\left(x_{t}\right)=\alpha_{l}\left(x_{t}\right)$ and $t \leq j$. Similarly, from $\alpha_{i}\left(x_{j}\right) \in \alpha_{l}\left(P_{t}\right)$, we see $\alpha_{i}\left(x_{j}\right)=$ $\alpha_{l}\left(x_{j}\right)$ and $t=j$. Choose $m$ smallest so that $c_{m j}=c_{i j}$ and choose $h$ smallest so that $c_{h j}=c_{l j}$. Then $\alpha_{i}\left(x_{j}\right)=\alpha_{m}\left(x_{j}\right)=x_{j}+m$, and $\alpha_{l}\left(x_{j}\right)=\alpha_{b}\left(x_{j}\right)=$ $x_{j}+h$; thus $h=m$ and $c_{i j}=c_{l j}=c_{l t}$.

We claim $\phi$ is an order isomorphism. If $c_{l t} \leq c_{i j}$ for some $0 \leq l, i \leq n$, $0 \leq t \leq k_{l}, 0 \leq j \leq k_{i}$, then since $X$ is a tree, $c_{l t}=c_{i t}$ and $t \leq j$. But $\phi\left(c_{l t}\right)$ $=\phi\left(c_{i t}\right)=\alpha_{i}\left(P_{t}\right) \cap R$ and $\phi\left(c_{i j}\right)=\alpha_{i}\left(P_{j}\right) \cap R$. Now $t \leq j$ implies $P_{t} \subseteq P_{j}$ and $\alpha_{i}\left(P_{t}\right) \cap R \subseteq \alpha_{i}\left(P_{j}\right) \cap R$. Thus $\phi\left(c_{l t}\right) \subseteq \phi\left(c_{i j}\right)$. This completes the proof.

Remarks. It is unknown whether the Bezout domains $R$ constructed here enjoy the property: every finitely generated $R$-module is a direct sum of cyclic modules. In [M1], Matlis shows the property is possessed by rings of the type we have constructed if each nonzero prime ideal is contained in a unique maximal ideal.

The procedure used in our construction can be used to produce certain infinite trees. For example, we can certainly construct a maximal valuation ring $\Theta_{0}$ such that spec $\Theta_{0}$ looks like $\mathbf{N}^{-1}=\{1 / n\} \cup\{0\}$, where $n$ ranges over the natural numbers. By altering this $\Theta_{0}$ as outlined above, we can display any tree (1) which has only finitely many maximal chains $C_{i}$, and (2) each $C_{i}$ looks like $\mathbf{N}^{-1}$.

In [L], Lewis shows $X \sim \operatorname{spec} R$ for some Bezout domain $R$ if and only if (1) $X$ has a unique minimal element; (2) if $x, y, z \in X$ and $x \leq z, y \leq z$, then $x \leq y$ or $y \leq x$; (3) if $x, y \in X$ and $x<y$, then there exists $z, w \in X$ with $x \leq z<w \leq y$ and no elements of $X$ lie between $z$ and $w$; and (4) 
every totally ordered subset of $X$ has a least upper bound and greatest lower bound. He shows that, for any partially ordered set $X$, there is a Bezout domain $R$ with spec $R$ order isomorphic to $X$ if and only if $X$ satisfies (1)(4). In his construction, Lewis makes use of the Bezout domains constructed by J affard which appear in [J, Theorem 3, p. 78] and [O, p. 586]. Jaffard's Bezout domain is an overring of a group algebra $F[G]$ with the same quotient field as $F[G]$, where $F$ is a field and $G$ is a lattice ordered group. Thus, it can be shown that the domains Lewis produces are not locally maximal, if the partially ordered set has more than one element. (See for example [S, p. 36, 51].) We have still not answered the following question in the infinite case: is there a locally maximal Bezout domain for any partially ordered set $X$ satisfying (1)-(4)?

Added in proof. The author has shown that if a domain $R$ has the property that every finitely generated $R$-module is a direct sum of cyclics, then every nonzero prime ideal of $R$ is contained in a unique maximal ideal.

\section{REFERENCES}

[J] P. Jaffard, Les systèmes d'idéaux, Travaux et Recherches Mathématiques, IV, Dunod, Paris, 1960. MR 22 \#5628.

[K] I. Kaplansky, Commutative rings, Allyn and Bacon, Boston, Mass., 1970. MR $40 \# 7234$.

[L] W. J. Lewis, The spectrum of a ring as a partially ordered set, J. Algebra 25 ( 1973), 419-434.

[M1] E. Matlis, Decomposable modules, Trans. Amer. Math. Soc. 125 (1966), 147-179. MR 34\#1349.

[M2] E. Matlis, Rings of type I, J. Algebra 23 (1972), 76-87. MR 46 \#5312.

[o] J. Ohm, Semi-valuations and groups of divisibility, Canad. J. Math. 21 (1969), 576-591. MR 39\#4146.

[S] O. F. G. Schilling, The theory of valuations, Math. Surveys, no. 4, Amer. Math. Soc., Providence, R. I., 1950. MR 13, 315.

DEPARTMENT OF MATHEMATICS, UNIVERSITY OF NEBRASKA, LINCOLN, NEBRASKA 68508 